\title{
NETs by-products and extracellular DNA may play a key role in COVID-19 pathogenesis: incidence on patient monitoring and therapy
}

\author{
Alain R. Thierry ${ }^{1 *}$ and Benoit Roch ${ }^{2}$ \\ 1* Research Institute of Cancerology of Montpellier, INSERM U1194, IRCM, ICM, Université de \\ Montpellier, Montpellier, F-34298, France \\ 2 Respiratory Medicine, University Hospital of Montpellier, Montpellier Université, Montpellier, \\ France. \\ *corresponding author: alain.thierry@inserm.fr
}

\begin{abstract}
Neutrophils play an important role as the first line of innate immune defense. One function of neutrophils, called neutrophil extracellular traps (NETs), has been discovered recently. NETs are extensive fibrous structures released extracellularly from activated neutrophils in response to infection. They are composed of cytosolic protein assembled on a scaffold of released chromatin. These structures suppress the dissemination of micro-organisms in blood by trapping them mechanically, and by exploiting coagulant function to segregate them within the circulation. In addition, NET components (DNA, histone, and granule proteins) also contribute to the triggering of an inflammatory process. NET function, however, can be regarded as a double-edged sword. On one hand, NET formation is an efficient strategy for neutralizing invading micro-organisms. On the other hand, NET can be harmful to the host, as its exposed by-products that are toxic to endothelial cells and parenchymal tissue. We present here the analogous biological and physiological features of the harmful positive amplification loop between inflammation and tissue damage induced by NETosis dysregulation and Coronavirus Disease-2019 (COVID-19) pathogenesis. Considering the rapid evolution of this disease symptoms and its lethality, we hypothesize that COVID-19 progresses under an amplifier loop, leading to an massive, uncontrolled inflammation process. We also describe the correlations of COVID-19 symptoms and biological features with those consecutive to uncontrolled NET formation causing various sterile or infectious diseases. General clinical conditions, and numerous pathological and biological features, are analogous with NETs deleterious effects. We postulate that Severe Acute Respiratory Syndrome-Coronavirus 2 (SARS-CoV2) induces a disproportionate virus-induced NET release, and that this plays a key role in COVID-19 pathogenesis. While neutrophils are the principal starting point for extracellular and circulating DNA release, targeting NETs rather than neutrophils themselves may stand for an effective strategy. This paper offers an in-depth review of NET formation, function and pathogenic dysregulation, as well as of current and future therapies to control NET unbalance. As such, it enables us also to suggest new therapeutic strategies to fight COVID-19. In combination with or independent of the latest tested approaches, we propose that, in the short term, deoxyribonuclease I (DNase-1) treatment should be evaluated; we also advocate a significant increase in research on the development of toll-like receptors (TLR) and C-type Lectin like receptors (CLEC) inhibitors, and on anti-IL26 therapies.
\end{abstract}




\section{Introduction}

To our knowledge, no other report describes COVID-19 pathogenesis in light of NETs formation and downstream effects. In this review, we focus on the possible link between COVID-19 pathogenesis and NET dysregulation. We include an overview of NET formation, function and pathogenic dysregulation, as well as existing and future therapies designed to control NET unbalance and its potential adverse effects. Because of the complexity of both COVID-19 and of NETosis, which involve a great number of pathways and signalings, we have arbitrarily described in detail the common biological and physiological features as currently understood. Our objective is to subsequently propose new strategies for treating the pandemic.

December 2019 saw the emergence of SARS-CoV-2 which causes COVID-19 (coronavirus disease2019), an acute respiratory disease (Wu et al, 2020). The Center for Disease Control and Prevention (CDC) reported illnesses have ranged from mild symptoms to severe illness and death for confirmed cases. Incubation lasts on average 5 days, with extremes of 2 to 12 days, the onset of symptoms appearing gradually with headache, muscle pain, fatigue/tiredness, fever, cough, shortness of breath and lethality. The WHO distinguishes several clinical syndromes associated with SARS-CoV-2: asymptomatic forms, uncomplicated disease, non-severe pneumonia and severe pneumonia, acute respiratory distress syndrome (ARDS), i.e. life-threatening respiratory insufficiency, sepsis and septic shock with multivisceral failure syndrome.

Infection-related conditions such as viral, bacterial, or mycotic pneumonia may directly injure lungs and cause acute lung injury (ALI) or ARDS. ALI is described as a lung disease with acute onset and disruption of the alveolar-capillary interface leading to increased microvascular permeability. ALI and ARDS can have many different causes, but epithelial injury is the basis of ARDS, and it is a more severe form of ALI. We propose to correlate ARDS/ALI with innate immunity in respect to COVID-19.

\section{Innate immunity}

All organisms constantly encounter invading microbes. Cells participating in innate immunity represent the body's first line of defense, being responsible for neutralizing invaders and steering the organism's adaptive immunity in case of persistent infection (Yousefi et al, 2019). Given the growing evidence of the part they play in antiviral immune responses, neutrophils can be said to be the main 'foot soldiers' in constituting such innate immunity.

The generally accepted dogma stipulate that neutrophils fight against micro-organisms by directly phagocytosing their targets, or by releasing toxic components via degranulation. It should be noted, however, that neutrophils also indirectly defend the host against microbes by participating in the elaboration of cell signaling networks involving cytokines, chemokines, survival and growth factors that cause downstream pro-inflammatory effects. Typically, neutrophils are found in higher concentrations in the pulmonary capillaries, as compared to systemic blood, even in the absence of inflammatory stimuli. This phenomenon allows neutrophils to readily migrate into the lungs in response to inflammatory stimuli. Neutrophils undergo cellular deformation in order perform a transendothelial migration from pulmonary capillaries to alveolar air spaces. Neutrophil activating factors may be derived from the host [e.g., platelet activating factor (PAF), leukotriene B4, interleukin-8 (IL-8)], or from pathogens [e.g., formylated peptide (fMLP) and lipopolysaccharide (LPS)].

Over the last 25 years, the importance of cell death pathways and the way in which immune cells respond to dead and dying cells has been intensively studied and its importance in many diseases demonstrated. In this context, an additional antimicrobial function of neutrophils should be noted, one 
which relies on a special type of programmed cell death called NETosis (Elkon et al, 2019). In response to injury, neutrophils are able to generate threads of chromatin covered with granule-derived peptides and (proteolytic) enzymes, namely neutrophil extracellular traps (NETs) (Pertiwi et al, 2017). These virus-induced NETs can control the virus by means of their large net-like structure in which the pathogens get trapped (Stiel et al, 2019).

\section{NETosis and extracellular DNA/nucleic acids release}

NETs were discovered in 2004 (Brinkmann et al, 2012), and have been described as a potential bacterial killing mechanism. Brinkmann et al. first reported that neutrophils were activated to release their DNA, which was laden with proteases that trapped and killed microbes (Pilscsek et al, 2010).

\section{NETosis/general function}

Described for the first time in 2004, NETosis constitutes a new effector mechanism of the neutrophil, allowing the release of NETs in the extracellular medium. These NETs correspond to extracellular filaments of uncondensed chromatin (an association of DNA and histones) covered by numerous proteins of mainly granular origin.

NETs have been linked with severe infections, such as sepsis, in which the release of chromatin decorated with neutrophil granular proteins serves as an additional defense of the innate immune system against circulating microbes, including bacteria, fungi, protozoa, and viruses. Pertiwi Conservation of the NET function across species suggests an evolutionary advantage of NETs in immune defense. VG The formation of NETs was originally described as a new cell death program, different from apoptosis and necrosis.

This "classic" NETosis is dependent on the oxidative explosion, and leads to the release of NET by 20 to $60 \%$ of human neutrophils, after 2 to 4 hours of stimulation with microorganisms or activators of protein kinase C (PKC), such as phorbol myristate acetate (PMA). During NETosis, the nuclei of neutrophils lose their characteristic shape, and chromatin decondensation takes place. During this process, the histones are cleaved by the granulation-derived-elastase from the granulations and citrullinated by peptidyl arginine deiminase 4 (PAD4), which leads to decondensation and sagging of the chromatin. Citrullination of histone $\mathrm{H} 3$ is thought to be involved in the formation of NETs. The combined rupture of the nuclear and granular membranes leads to the mixing of the cytoplasmic, granular and nuclear components. The rupture of the plasma membrane then allows the release of NETs in the extracellular space. Initially, neutrophils elastase degrades the linker histone protein $\mathrm{H} 1$ and the core histone protein, resulting in chromatin decondensation, which is enhanced by myeloperoxidase (MPO). The main components of NETs are deoxyribonucleic acid (DNA) and histones $\mathrm{H} 1, \mathrm{H} 2 \mathrm{~A}, \mathrm{H} 2 \mathrm{~B}, \mathrm{H} 3$, and $\mathrm{H} 4$; other components which have been reported include: neutrophil elastase, MPO, bactericidal/permeability-increasing protein, cathepsin G, lactoferrin, matrix metalloproteinase-9, peptidoglycan recognition proteins, pentraxin, and LL-37 (Hirose et al, 2014).

\section{$\underline{\text { Stimuli }}$}

The stimuli inducing polynuclear neutrophils (PN) PN (Polynuclear neutrophils) NETosis varies. It includes many micro-organisms and their components (LPS, lipopolysaccharide), pro-inflammatory cytokines [IL-8, tumor necrosis factor $\alpha$ (TNF $\alpha$ )]), activated platelets or immune complexes (Yeng et al, 2018). The molecular mechanisms leading to NETosis have yet to be fully established. However, various studies have given rise to a "classic" model involving PN cell death, as well as "alternative" models. Thus, there would appear not be one but several mechanisms of NETosis, depending in particular on the trigger stimulus.

In the 'classic' model, modification by citrullination of histone H3 is thought to be involved in the in vitro formation of NETs. Hirose et al (2014) have described the relationships between the presence of NETs and citrullinated histone $\mathrm{H} 3(\mathrm{Cit}-\mathrm{H} 3)$ with the existence of bacteria in tracheal aspirate, SIRS, 
diagnosis, white blood cells (WBC) count, and concentrations of IL-8, TNF-a, cirDNA, lactate, and high mobility group box-1 (HMGB1). NET-inducing factors identified to date include IL-8, PMA, bacteria, mycobacteria, fungi, protozoa, PAF, LPS, and M1 protein. Classic NETosis requires the generation of ROS by NADPH oxidase. However, mitochondrial ROS production in the absence of a functional NADPH oxidase is sufficient to trigger NETosis. ROS act as key signaling molecule for NET formation independent of the stimulus and of the type of granulocyte. In order to maintain homeostasis, NETs are then degraded by the combined action of DNase- 1 and an endocytosis mechanism involving macrophages.

In counterpart to this "classic" model, several studies have reported a phenomenon of rapid NETosis (5-60 $\mathrm{min}$ ) in different experimental models. The cellular mechanism is variable, but none of these rapid NETosis cause the rupture of the plasma membrane, nor the death of the PN. Some involve the release of mitochondrial DNA (stimulation by the association of $\mathrm{C} 5 \mathrm{a}$ [complement protein $5 \mathrm{a}$ ] and GMCSF [granulocyte-macrophage colony-stimulating factor]. Others extracellularly release vesicles containing nuclear DNA (stimulation by S. aureus). A recent study even showed that, after NETosis, PN retain their ability in migrating and in performing phagocytosis. This process is asociated by the release of neutrophil-derived enzymes. This alternative rapid mechanism would explain the role of NETosis in certain acute phenomena (Granger et al, 2014).

\section{Role of platelets}

Neutrophils and platelets have a ball dance in potentializing an innate immune response. NETosis involves platelets being sequestered in the lung microcirculation in a neutrophil-dependent process. In preclinical studies, Caudriller et al (2012) have shown that platelet depletion protects mice from severe lung injury and mortality. Platelets promote inflammation and injury through critical interactions with neutrophils. For instance, in sepsis, the activation of platelet TLR4 is a potent stimulus for the release of NETs by neutrophils residing in the sinusoids of the liver and in other capillary beds They observed ultrastructural evidence of neutrophil-platelet aggregates in the lung microcirculation which included close interactions with the lung endothelium and red blood cells. The innate immune postulated function of NETs resides in skimming plasma for intravascular pathogens; other intravascular components, nonetheless, may also be efficiently trapped, causing detrimental effects. Notably, an intense platelet sequestration in areas of NET formation was observed (Caudriller et al, 2012. The high binding of platelets to NETs is is mediated through interactions with charged extracellular histones. Platelet accumulation on NETs may perpetuate platelet aggregation and activation, promoting coagulation in the lung microcirculation, which may potentially lead to ischemic consequences.

\section{NET and extracellular DNA release function: a double-edged sword}

On one hand, as an innate response, NET formation is an efficient strategy for strategy for offsetting invasive micro-organisms such as bacteria and fungi. On the other hand, NET can have harmful effects on the host, as its exposed compounds are toxic to endothelial cells and to parenchymal tissue (Yeng et al, 2013).

\section{Beneficial innate response}

The anti-infectious role of NETs, as a new effector mechanism of innate immunity, has been widely documented since their discovery. The original structure of NETs allows them to capture a wide variety of microorganisms; thus, many examples of target bacteria for NETosis appear in the literature, such as S. aureus or Mycobacterium. tuberculosis. It has also been shown that fungi (Candidaalbicans, Aspergillus sp.), Protozoa (Toxoplasma gondii) and, more recently, viruses like human immunodeficiency virus (HIV), are sensitive to the antimicrobial action of NETs.

Evidence is accumulating that neutrophils play a role in antiviral immune responses. These virusinduced NETs can both control the virus and damage the host (Schonrich et, 2016) . 
In vitro experiments have indicated that viruses can directly stimulate neutrophils to produce low levels of NETs (Schonrich, 2016). For example, neutrophils sense HIV-1 by endosomal PRRs (pattern recognition receptors) that detect viral nucleic acids, and mediation of to TLR7 and TLR8 subsequently induces NETosis (i.e. influenza virus A). Viruses also induce NETosis indirectly without engaging PRR expression by neutrophils. Thus, NETosis is triggered by the cytokines and chemokines, such as IL-8, contained in the inflammatory milieu created by virus-infected endothelia and epithelia. In addition, type I interferon (IFN) is produced abundantly during viral infections and it primes neutrophils to form NETs. Growing evidence suggested that platelets are important for defense against viruses, as platelet are frequently activated during viral infection. For example, single-stranded RNA viruses from the family Picornaviridae family activate platelets through TLR7, reducing viral titers and increasing host survival. Activated platelets aggregate with neutrophils and thereby stimulate NETosis, as reviewed by Schonrich et al. Massive activation of the platelet/neutrophil axis and subsequent NET-based clearance mechanisms may represent a host emergency strategy when facing systemically multiplying viral infections.

Alternatively, there are direct mechanisms by which NETs develop antiviral activity. First of all, the web-like chromatin backbone of NETs can bind to, and immobilize, viral particles due to the attraction between positively charged histones and the negatively charged viral envelope. This mechanically prevents the virus from spreading. Examples include histone $\mathrm{H} 1$ binding to noroviruses and $\mathrm{H} 3$ and $\mathrm{H} 4$ aggregation of seasonal influenza A particles. Second, known antimicrobial molecules against both enveloped and non-enveloped viruses, such as MPO, cathelicidins, and $\alpha$-defensin, are attached to the chromatin backbone of NETs and can inactivate viral particles. However it is less clear how NETs contribute to antiviral immunity.

In addition, NET components indirectly contribute to antiviral immunity by stimulating antiviral effector mechanisms from other immune cells. For instance, histones and high mobility group box-1 (HMGB1) proteins anchored in NET fibrin act as danger-associated molecular patterns (DAMPs) that trigger release of pro-inflammatory cytokines and chemokines by other immune cells. Moreover, neutrophil-derived NETs build aggregates that in turn degrade cytokines and chemokines, resulting in self-limitation of the innate immune process. In addition, NETs activate plasmacytoid dendritic cells (pDCs) through TLRs. NETs thus play a key role in antiviral immunity by releasing large amounts of type I IFN. Additionally, NETs can be enriched in oxidized mitochondrial DNA, which is efficiently induces a type I IFN response.

\section{Detrimental innate response: Pathologies induced by unbalanced NETosis}

Below, we have arbitrarily described inflammatory diseases, viral pathogenesis and respiratory illnesses relevant to COVID-19.

\section{Sterile Pathologies}

NETs are not produced exclusively during severe infections. They have also been observed in inflammatory diseases such as pre-eclampsia, small-vessel vasculitis, and systemic lupus erythematosus (SLE). NETs are also found in noninfectious diseases in which the presence of NETs may be a maladaptive response leading to tissue injury. These sterile pathologies induced by unbalanced NETosis provide insight into the pathogenesis of COVID-19.

\section{Autoimmune NET vs SLE}

The deleterious role of NETs has been particularly studied in the context of autoimmune diseases, especially lupus. NETs expose many autoantigens in the extracellular space, in particular double strand DNA or MPO. Numerous studies have demonstrated the existence of the deregulation of NETs during lupus. The immune complexes found in the sera of patients suffering from SLE typically contain nucleic acids associated with various proteins. These include antibodies, the chromatin-associated protein HMGB1, the antimicrobial peptide LL39, ribonucleoproteins, and others (Lamphier et al, 2014). 
Patients with Lupus have increased circulating levels of NETs, and evidence for NET formation can also be found in those patients' tissue. There is some evidence that patients with SLE or vasculitis have an impaired ability to degrade NET (Elkon et al, 2019).

The increase in NET production is triggered by the presence of high concentrations of proinflammatory cytokines (IL-18, IFN] [interferon [?]). At the same time, the deterioration in the degradation of NETs identified in certain patients is due to those same NETs protecting themselves against DNase-1. They do so by the binding of autoantibodies and / or of the complement to their surface. This protection by steric hindrance leads to an accumulation of NET, which is in turn responsible for a chronic stimulation of the pDCs via TLR9. The consequence is a continuous release of IFN?], a key cytokine in the pathophysiology of lupus. This cytokine is also responsible for sensitizing PNs to NETosis. The overall result is the establishment of an amplifier loop which could participate in the sustainability of the autoimmune reaction and the progression of the disease.

\section{Thrombosis}

An increasing number of studies demonstrate the essential role of NETs in thrombotic pathologies, both venous and arterial. The procoagulating effect of NETs underpinned by their filament structure, which constitutes their structure in a filament network, which facilitates adhesion and platelet activation. This structure also allows them to capture red blood cells, as well as various coagulation factors, enabling the formation of the thrombus and the initiation of coagulation. NETs also activate the intrinsic and extrinsic pathways of coagulation indirectly, by the action of serine proteases (elastase and cathepsin G) on coagulation inhibitors, but also directly, since the NET nucleic acids activate factor XII (FXII) and that, in certain pathologies (sepsis, vasculitis), NETs express tissue factor on their surface, thus enabling activation of the extrinsic pathway of coagulation (Moschonas et al 2019.

Recent studies in mice suggest that DNA and histones are released during inflammation, or infection, and that this stimulates coagulation, thrombosis, thrombocytopenia, and organ damage (Fuchs et al, 2012). Fuchs et al (2012) observed that nucleosomes and inflammatory markers are concomitantly elevated in acute thrombotic microangiopathy (TMA) and that these molecules reflect the disease state. The authors identified inflammation markers, namely, myeloperoxidase (MPO) in neutrophil granules and the abundant S100A8/A9 hetero-complex from neutrophil cytoplasm. They postulated that inflammation, in response to even a minor infection, might result in the generation of extracellular DNA and histones and thus trigger acute disease in patients at risk of TMA. Jimenez et al (2015) have reported that low levels of DNase1 lead to an impaired NET degradation in vitro; in patients reduced plasma DNase-1 activity leads to prothrombic NET accumulation (Kumar et al, 2018). Pertiwi et al (2018) demonstrated that neutrophils and NETs are intricately involved in all the types of acute haemorrhagic or thrombotic plaque complications, and that the NETs disperse as the thrombus mass ages.

\section{Coagulation}

Neutrophils may play a major role in immunothrombosis, which is defined by interactions between innate immunity, inflammation and thrombosis, leading to thrombin generation, especially in microvessels. Furthermore, cytokines released by neutrophils can alter the membrane profile of vascular cells, especially endothelial cells (Stiel et al, 2019). These cytokines thus participate in coagulation activation and septic shock-induced endothelial dysfunction. McDonald et al (2018) demonstrated that NETs promote intravascular coagulation in mice; and Stiel et al (2019) first visualized circulating NETs during septic shock-induced disseminated intravascular coagulation. Intravascular NETs may induce platelet trapping and microvascular occlusion leading to multi-organ failure.

\section{Inflammatory disease}

It is likely that the formation of NET during acute and chronic inflammatory diseases is favored by tissue- and systemic- proinflammatory cytokines. During sepsis, endothelial damage, tissue 
ischemia, and NET-induced thrombotic complications are implicated in multiple organ failure and increased lethality. More specifically, in experimental models of sepsis, NETs accumulate in the pulmonary and hepatic microcirculations, leading to acute hepatocellular insufficiency and to acute respiratory distress syndrome by hypoperfusion. In addition, in humans the presence of NET has been reported in blood samples from approximately half of patients with severe sepsis. In acute inflammatory lung diseases such as ARDS or post-transfusion acute respiratory syndrome, activated platelets induce the formation of NET, which accumulate at the alveolar level and participate in epithelial and endothelial lesions (as reviewed by Cheng et al, 2013). In fact, it has been shown that histones are capable of inducing dose-dependent cell apoptosis, and that elastase increases the permeability of the alveolocapillary membrane by alteration of the endothelial cytoskeleton. During post-transfusion acute respiratory syndrome sepsis, circulating markers of NETosis are present.

During pre-eclampsia, certain placental factors (i.e. IL-8) are capable of inducing NET formation in vitro. Ex vivo, NETs are abundantly found in the intervening space of the placentas, and may contribute to the hypoxic lesions characterizing this pathology. In gout, it has been shown that sodium urate crystals are capable of inducing the formation of NET, and that these are protected from degradation by the fixation of C-reactive protein (CRP) molecules and of the complement (Granger et al, 2014). NETs could therefore persist long enough at the joint to contribute to the establishment of chronic inflammation. In cystic fibrosis, several studies have demonstrated the abundant presence of NET in the sputum of patients. The bacteria commonly found in these patients (Pseudomonas aeruginosa, S. aureus and Haemophilus influenzae) are good inducers of NETosis, but are also capable of acquiring mechanisms of resistance to NETs, thus promoting bacterial colonization and participating in lesions of the pulmonary epithelium via elastase. Recently, NETs have been demonstrated in histological sections of tumors; they mayexert a protumoral or anti-tumor effect, depending on the type and stage of the cancer or even the tumor microenvironment. The impact of NETs on the organism becomes more complex as knowledge of these structures develops.

In addition to their anti-infectious role and their various deleterious aspects, therefore, it appears likely that NETs also exercise a regulatory function. Barrientos et al (2014) developed a NET isolation and purification protocol enabling to show an immuno-regulating role of NETs on activated dendritic cells.

\section{Transfusion TRALI}

Blood transfusions are one of the well-recognized causes of ALI. Transfusion-related ALI (TRALI) is the leading cause of death from transfusion therapy in the US, and a major cause of transfusion morbidity. While it is known that activated platelets induce the formation of NETs in TRALI, its pathogenesis nonetheless remains poorly understood. Caudrillier et al (2012) have shown that NETs increase endothelial permeability and are present in abundance in the lungs of mice undergoing experimental TRALI. They have also shown that NETs and circulating NET components are strongly increased in clinical cases of TRALI.

\section{Diabetes:}

Kumar Under diabetic conditions, neutrophils generate abundant cytokines and superoxide. The hyperglycemia can induce NET formation in type 2 diabetes patients. Hyperglycemic conditions cause oxidative stress and constitutively activate NETosis, which affects the normal immunological balance and also creates microvascular complications (Kumar et al, 2018).

\section{Sepsis}

Disseminated intravascular coagulation (DIC) is a severe complication of septic shock. Polymorphonuclear neutrophils (PMNs) may play a key role in septic shock-induced DIC via the release of NETs (Stiel et al, 2019. NETs capture invading pathogens, but also act as a pro-coagulant surface at the interface between immunity and thrombosis. During septic shock-induced DIC, neutrophil activation may lead to excessive NET formation. In patients with sepsis, circulating cell-free nucleosomal DNA correlated with morbidity and mortality. 
Others: Neutrophil extracellular trap formation and neutrophil activation may play a role in the pathogenesis of painful sickle cell crisis and acute chest syndrome. Elevated levels of circulating nucleosomes and neutrophil activation in sickle cell patients with painful crisis suggests NET formation in these patients. NETs are also involved in some non-autoimmune pathologies, such as cystic fibrosis, or periodontitis. Some recent studies suggest NET involvement in the physiotherapy of other autoimmune diseases, such as type 1 diabetes (Schimmel et al, 2013), autoimmune vasculitisrare conditions affecting small blood vessels, particularly those of the lungs, skin and kidneys, rheumatoid arthritis (RA) (Bach et al, 2020), Felty syndrome, or psoriasis. Formation of NETS was observed in pathologies sucha s liver diseases (alcool-associated liver disease and portal hypertension), severe obesity ( D'abbondanza et al, 2019), pre-eclempsia (Moodley et al, 2020) or kidney diseases (Nakazawa et al, 2018).

\section{Conclusion on NETs adverse effects}

Circulation of NETs in detectable amounts in the serum may impair or overwhelm the NET degradation and clearance machinery. This systemic NET 'overflow' has severe direct and indirect harmful effects. First, NETs can directly damage the endothelial cells lining the interior face of blood vessels. Second, NET overflow drives auto-destructive processes, as components of NETs act as neo self-antigens and induce autoantibodies. In view of this, a number of molecules that have been identified as important targets in autoimmune diseases (e.g., dsDNA, histones, MPO, vimentin, and enolase) are actually NET constituents.

These highly immunogenic NET structures generate a self-perpetuating cycle of autoimmune combat as the result of autoantibody production, further neutrophil recruitment and triggering of NETosis. This is in line with the characteristics of inflammatory lung diseases: exaggerated neutrophil recruitment, activation, and NET formation. The prolonged presence of NETs appears extremely deleterious to host tissue and can stimulate autoimmune responses due to its high immunogenicity. Although NET is principally responsible for physically clearing the virus when infection occurs, it triggers an auto-immune response, sometimes with severe adverse effects. This highlights NET's immunogenicity, which can lead most noticeably to respiratory deficiency, also observed in microbial infection.

\section{Microbe-induced Respiratory illness}

The respiratory tract is considered one of the most vulnerable places for microbial invasion of the body. In this context, it is possible that NETs are initially produced in response to pathogens before infection. Working on experimental TRALI, Caudriller et al (2012) have hypothesized that NET formation occurs in the lungs and is driven by interactions between activated platelets and neutrophils. NETs may produce lung endothelial injury mediated by exposed extracellular histones, neutrophil granular proteins, and by a tangled web of extracellular DNA. This may provide a template for the trapping of platelets and for thrombus formation in the lung microcirculation. NETs induce injury to the lung by a direct toxic effect on endothelial cells; this toxicity may be the result of a high local concentration of histones and granular proteins The observation of NETs in the lung microvasculature is associated with an increased presence of NET components in the plasma (Caudriller et al, 2012).

With regards to viral infection, NETs have been detected in the broncho alveolar lavage fluid of children with severe respiratory syncytial virus (RSV) infection of the lower respiratory tract. NETs were also detected in dense plugs occluding the small airways in RSV-infected calves. In a mouse model of influenza pneumonia, NET formation was also observed in areas of alveolar-capillary damage in the lung. Hamaguchi et al (2013) have shown that, in response to respiratory infection, neutrophils released NETs abundantly. Their regression analysis also indicated that NET length correlated with six 
clinical parameters (white blood cells, platelets, lactate, CXC ligand-2, interleukin-8, and procalcitonin) as the explanatory variables.

\section{NET and Viral pathogenesis}

As introduced earlier, unbalanced NET formation is associated with pathological conditions and is directly cytotoxic to epithelial and endothelial cells, as well as to hepatocytes. NET formation represents a plausible link between viruses and systemic autoimmune disease.

Note, transient systemic NET overflow, due to increased NET formation, without noticeable deficiency in DNase-1 activity, can occur during infection with hantaviruses. Neutrophils play an antiviral role during viral haemorrhagic fevers (VHF) caused by hantaviruses. In their elegant review, Schonrich et al. (2016) describe how these zoonotic pathogens belong to the family Bunyaviridae and infect humans after transmission via inhalation of aerosolized urine, saliva, and feces from chronically infected rodents, their natural hosts. In humans, they can induce severe pulmonary and renal dysfunction as well as intravascular coagulation and hemorrhagic shock. High levels of circulating NETs, and accordingly increased amounts of cirDNA and histones, are detected in hantavirus-infected patients. Thrombin generation, intravascular coagulation and increased endothelial barrier permeability feature among other hantavirus infection effects. These are in line with potential detrimental effects of excess of NET formation. Finally, NETs can induce autoantibodies that may contribute to the systemic pathology of hantavirus-associated disease. Neutrophils can detect HIV-1 via interaction with TLRs (TLR7 and TLR8) (as reviewed by Kumar et al, 2018). These TLRs recognize viral nucleic acids and induce the generation of reactive oxygen species by MPO-derived oxidants that trigger NET formation and elimination of HIV-1. This response may, however, be counteracted by HIV-infected dendritic cells that release, an anti-inflammatory cytokine, IL-10, which, in turn, inhibits NET formation. Notably, it was also demonstrated that, viral infections, such as RV and RSV, alter DNA methylation and histone modifications in the airway epithelium which may in turn alter inflammatory responses, exacerbating chronic airway inflammatory diseases (Tan et al, 2020). Furthermore NETs and neutrophils are involved in the pathologies of chikungunya virus (CHIKV), simian immunodeficiency virus (SIV), Influenza, Parvovirus, rhinovirus and influenza pneumonia.

\section{NET nucleotidic components: nucleosomes and circulating cell-free DNA}

\section{Nuclear DNA}

Before the discovery of NETs (Brinkmann et al, 2004), several studies reported an increase in the concentration of cirDNA in the blood of patients suffering from various diseases (Thierry et al, 2016). This discovery led to growing interest as to its potential use in various clinical fields. CirDNA analysis is currently applied in prenatal diagnosis and in oncology. The other fields in which it shows potential for clinical use include organ transplant, autoimmune diseases, trauma, myocardial infarction, diabetes, thrombosis and sepsis. CirDNA concentration has been shown to be greatly increased in cancer subjects, and significantly increased in the pathologies cited. It may also increase following intense effort. We (Sanchez et al, 2018) and others (Jiang et al, 2017; Underhill et al, 2018) revealed that cirDNA size profile shows a characteristic nucleosome fragmentation pattern. Our recent observations revealed that high molecular weight cirDNA fraction in the plasma of healthy individuals is minor, and corresponds to approximatively $12 \%$ of the total cirDNA fragment number (unpublished data). Thus, the proportion of cirDNA associated with mono-nucleosome (or to a much lesser extent with Transcription Factor complexes) circulating in blood is preeminent. This suggests that detectable cirDNA resident in plasma is predominantly associated with both structures (Snyder et al, 2016) The observed chromatin organized structures correspond to the chromatosome tightly associating 166 base pair (bp) DNA and constituted of a histone octamer (( $\mathrm{H} 2 \mathrm{~A}-\mathrm{H} 2 \mathrm{~B}) 2(\mathrm{H} 3-\mathrm{H} 4) 2)$ plus the histone 
monomer linker H1. Our data showed that the cirDNA molecule is highly nicked (97-98\%), and that continuous nuclease activity occurs on the nucleosomal structure. Thus, the highly represented among the cirDNA structural forms consist in a nucleosome structure corresponding to the chromatasome devoid of the histone monomer linker $\mathrm{H} 1$ as well as to the mononucleosome compacting only $147 \mathrm{bp}$ DNA is

The nucleosome appears as the most stabilizing structure of DNA in the circulation. cirDNA molecules, which are initially packed in chromatin, are released from cells and then dynamically degraded in blood. This occurs both within and between nucleosomes or transcription factor-associated subcomplexes. CirDNA or mononucleosomes in blood may be associated with a diversity of other structures. These range from tightly-packed long dsDNA, long-sized and short-sized DNA-associated micro-particles, apoptotic particles, short-sized lipoproteonucleic complexes, and cell or cell-part associations. Consequently, we hypothesize that cirDNA structure diversity results from different biological phenomena, which include: various cellular mechanisms of release, dynamic chromatin degradation in the circulation, and potential association with blood constituents. Given the high DNA sequence instability towards nuclease attack, only cirDNA protected by stable structures can be detected and residing in the blood stream. Nuclear cirDNA fragmentation results from mapping locations of the chromatin organization along the genome, which protect/packed DNA with mononucleosomes as the lower unit (Sanchez et al, 2018). For more than two decades, it was postulated that the prominence of mononucleosomes suggested apoptosis as the main mechanism of release. However, short-sized nucleosomal structures could also result from the progressive nuclease degradation of longer cirDNA originating from necrosis, phagocytosis, microparticle-containing DNA, or active release from lymphocytes, in particular NETosis. Indeed, chromatin fragments, oligonucleosomes and nucleosomes can be liberated from the degradation process of NETs, and contribute to the pool of cirDNA and histones.

Extracellular and intracellular self-DNA produced consecutive to any of the various cell death processes are rapidly degraded by various DNases, as well as aberrant self-DNA present in cytosol and endosomes. This clears cellular debris and prevents activation of self-DNA in normal physiological conditions. By itself, the accumulation of extracellular self-DNA would not be sufficient to trigger inflammation. In vitro and in vivo studies have revealed that even large amounts of extracellular DNA as well as NETs fail to stimulate immune cells.

Many studies have shown that, in certain conditions, both NETs and cirDNA (or Plasma DNA) may induce an inflammatory response. NETs contain numerous compounds which either have or induce the inflammatory properties presented above. In addition to the NET steric physical properties, this largely distinguishes NETs from mononucleosomes in respect to their inflammation and immunologic properties. Despite numerous studies, it remains uncertain that the global inflammatory response results from only one or other of these two entities. We believe it difficult or nearly impossible to fully discriminate both, following the induction of NETosis in an "in vivo" setting, given the rapid and significant activity of endo- or extra-cellular nucleases. As a consequence, we propose to combine the NETs and degradation products altogether under the term NETs in many aspects of this review.

However, the re-entry process of self-DNA in host cells is a biological feature that clearly differentiates NETs and mononucleosomes. (This process has been well reviewed by Poli et al, 2017) Nucleic acids appear to be the main source of DAMPs, and stimulate Pattern Recognition Receptors (PRR) to induce inflammation (Elkon et al, 2019). The location of nucleic acid sensing receptors can be conveniently divided into endosomal and cytosolic. They are recognized by DNA sensors such as TLR9, and by stimulator of IFN genes (STING), which are secluded in endosomes and cytosol, respectively; this recognition triggers type I IFN and proinflammatory cytokine secretion, thereby controlling host defense countermeasures. TLR9 is mainly expressed by B lymphocytes and pDCs in humans, and recognizes DNA containing unmethylated CpG motifs which are more prevalent in microbes. STING, expressed in numerous cell types (including myeloid and lymphoid cells) $(2,3)$, recognizes a wide variety of DNA. Nucleic acids, and especially short pieces of DNA (mainly in the form of mononucleosomes in the circulation, constitute potent microbial moieties that activate innate immune cells. CirDNA in the form of nucleosomes has been reported to correlate with organ 
dysfunction, disease severity and mortality in sepsis patients and children suffering from meningococcal sepsis.

Under pathologic conditions, extra-cellular DNA and histones may be part of a feedback-loop between inflammation and tissue damage. Necrosis attracts neutrophils that in turn exacerbate tissue damage. In vitro experiments suggest that NETs, mononucleosomes or extracellular histones contribute to the cytotoxicity (Fuchs et al, 2012)._Holdenrieder et al (2012) first demonstrated the role of nucleosome in autoimmune disease and other authors showed that histones are antimicrobial, yet can also cause tissue damage and other pathological abnormalities. Thus, histones released from NETs represent, by itself, unique cytotoxic DAMPs linking cell necrosis and inflammation (as reviewed by Nakasawa et al, 2018).

\section{Mitochondrial DNA}

Infections by bacteria, fungi, and parasites causes release of some nuclear DNA and abundant mitochondrial DNA (mtDNA). Various studies provided convincing evidence for the role of mitochondria in NET formation and their possible role as a DAMP. Yousefi et al. ( 2019) identified mitochondria as a source of scaffold DNA for NETs. Interestingly, all types of granulocytes, neutrophils, eosinophils, and basophils, release mtDNA to form extracellular traps, which then bind and kill bacteria in the absence of detectable cell death. In addition, oxidized mtDNA is highly inflammatory as it activates the STING pathway, inducing IFN-b and other inflammatory cytokines (Elkon, 2019). Mitochondria are readily damaged through hyper- or hypo-polarization of their membranes and by generation of ROS. Elkon et al (2019) demonstrated that once oxidized, mtDNA is less efficiently degraded, by DNases such as TREX1, leading to activation of DNA sensors (An et al, 2018).

Additionally, several investigators have observed that mtDNA is released in the absence of neutrophil death.There are several ways mitochondria can impact immunity especially innate immunity. For example mtDNA is rich in CpG DNA (potential TLR9 ligand) and can gain entry into cells through binding to Tfam. Furthermore, ROS oxidizes mtDNA, apotential cGAS ligand, that cannot be efficiently repaired within the organelle. Finally, because of its bacterial ancestry, mitochondrial peptides have an $\mathrm{N}$ terminal formylated methionine that stimulates the production of chemokines. Overall then, mtDNA appears more like "foreign" than "self" DNA (Boudreau et al, 2014). As such, mtDNA is a highly potent inflammatory trigger and is reportedly found outside the cells in blood in various pathologies. Yousefi et al (Yousefi, 2019)speculated that positively charged granule proteins that are released together with mtDNA act as building blocks connecting one mtDNA molecule to another, building a fiber-like mtDNA net. There is increasing evidence that NETs are generated by viable neutrophils releasing mtDNA and granule proteins.

Boudreau et al (2014) hypothesized that activated platelets could release their mitochondria, both within membrane-encapsulated micro-particles and as free organelles. They found extracellular mitochondria at higher levels in platelet concentrates of TRALI patients experiencing acute reactions like febrile non-hemolytic reactions, skin manifestations, and cardiovascular events. Note, we recently demonstrated the presence of circulating cell-free respiratory competent mitochondria in blood of healthy individuals without any particular activation (Al Amir Dache et al, 2020). One can think that they might contribute to the total amount of mitochondrial components in blood stream.

As recently reviewed by Tiku et al (2020), viruses appears to influence microbial pathogenesis by manipulating mitochondria or their constituents. Several viruses (Dengue virus, HIV and SARS) induce mitochondrial fusion that is required for intracellular proliferation of the virus and evasion of the antiviral innate immune signaling. Moreover, open reading frame 9b (ORF-9b), a virulence factor of SARS-CoV, induces proteasomal degradation of dynamin-related protein 1 (DRP1), thereby leading to mitochondrial fusion and fragmentation, which eventually limits host cell ilFN responses against the virus (Shi et al, 2014). Injecting purified human and murine mtDNA into mouse joints led to arthritis in mice. This effect was not observed with nuclear DNA, revealing the inflammatory nature of mtDNA. Furthermore, mtDNA and the mitochondrial lipid cardioliopin act as potent activators of the NLRP3 
inflammasome. NOD-Like Receptors (NLRs) activate inflammasomes, which are multiprotein complexes that act as platforms for the activation of proinflammatory caspase-1. Here, pathogen recognition receptors (PRRs) are the cytosolic nucleotide-binding oligomerization domain (NOD)-like receptors (NLRs). With regard to the innate immune response, mtDNA present in the cytosol is sensed by cGAS, and the cGAS-STING pathway is turned on and generates a downstream immune response driving type I IFNs and other proinflammatory cytokines.

Tiku et al (2020) described the effect of infection on mitochondrial dynamics, as bacteria and viruses induce changes in mitochondrial morphology. Influenza A leads to the dissipation of mitochondrial membrane potential, which ultimately causes mitochondrial fragmentation. Dengue virus (DV) inhibits DRP1 and induces mitochondrial fragmentation, which is necessary for its replication and immune evasion. Thus, targeting mitochondria seems to be a hostimmunity evasion mechanism of the pathogen. It is conceivable that this might lead to mtDNA alterations, release into cytosol or outside the cell, eliciting a counterbalancing inflammatory response by the cell as described above.

The exact mechanism whereby mtDNA can escape to the cytosol remains unknown. At this stage of research it is difficult to clearly gather the role of the virus intracellular targeting of mitochondria from the role of the extracellular mitochondria components release.

\section{RNA and modified nucleic acids}

Our understanding of the rules for distinguishing self from foreign by innate immune sensors is at an early stage. For RNA, $5^{\prime}$ capping of the terminus is an excellent example, as this feature is present in many viruses, but not host RNA. As highlighted by Elkon et al (2019), RNA-reactive TLRs can detect both virus and mammalian RNA. But as usual, sequence and structure are most likely key determinants of ligand avidity, as TLR7 and TLR8 preferentially bind unmodified uridine (U)-rich ssR (Elkon et al, 2019). Tan Epigenetic modifications, such as DNA methylation, may also exacerbate chronic airway inflammatory diseases. DNA sequence is still relevant because mammalian DNA sequences enriched for CpG dinucleotides are more potent activators of TLR9 than DNA sequences devoid of CpG dinucleotides (Tan et al, 2020). CpG islands, mtDNA, and retroelements are potential sources of immuno-stimulatory DNA in mammals. Double-stranded RNAs were shown to activate nucleic acidrecognizing TLRs, including TLR3; TLRs 7 and 8 are activated by single-stranded RNAs. Note, NET formation is stimulated by exposure of neutrophils to certain bacteria, immune complexes (ICS) containing RNA which, in turn stimulates the intracellular TLR8, the dominant TLR in neutrophils (Elkon et al, 2019).

\section{DNA re-entry into cell}

In chronic inflammatory disorders, exacerbated inflammation has been associated with a break in innate immune tolerance to self-DNA. In physiological conditions, self-DNA released by dying cells is not detected by intracellular DNA sensors. Excessive nucleic acids released by necrosis or NET may reenter the cell. Certain types of nucleic acids can provoke a robust innate immune response, and this recognition is mediated by cytoplasmic receptors, such as retinoic acid-inducible gene 1 , and by TLRs localized inside endosomes and lysosomes (Elkon et al, 2019). The TLRs recognize a wide array of pathogen-associated and endogenous molecular patterns that trigger innate immune responses (reviewed in Sasai and Yamamoto, 2013). TLRs can be divided into two categories, those that are mostly expressed on the cell surface (such as TLR2 and TLR4) and those expressed predominantly in the intracellular compartments, endosomes and lysosomes. TLRs are central to the control of innate immune responses through the recognition of pathogen-associated molecular patterns (PAMPs) and endogenous DAMPs (Lamphier et al, 2014). Therefore, TLRs are not only critical for host defense against pathogens but they also contribute to the pathogenesis of autoimmunity. These associated proteins may protect the bound nucleic acid from degradation and/or facilitate their entry into the cell, as is the case for Fc receptor-mediated uptake of antibody-nucleic acid complexes. TLR-mediated pathologic responses to nucleic acids may contribute to other pathologies, such as damage due to liver injury or lung infection, pancreatitis, and graft-versus-host disease. 
Consequently, re-entry capacity makes nucleic acids as appearing "ectopic" and are seen as foreign invaders and activate nucleic acid sensors (reviewed in Elkon et al). For instance, re-entry in SLE was identified as being through IgG autoantibodies that form immune complexes with nucleoprotein particles and engage the activating Fc gamma receptor, FcygRIIA on pDCs and other cells. This in turn led to phagocytosis and downstream activation of intracellular TLRs, FcgRIIA on pDCs and other cells. This in turn led to phagocytosis and downstream activation of intracellular TLR to stimulate the production of IFN-I and inflammatory cytokines.

Poli et al discovered that IL-26 binds to genomic DNA, mitochondrial DNA, and neutrophil extracellular traps, and that it shuttles them in the cytosol of human myeloid cells. IL-26 is a member of the IL-10 cytokine family, overexpressed in numerous chronic inflammatory diseases, in which its biological activity remains unclear. IL-26 overexpression allows extracellular DNA to trigger proinflammatory cytokine secretion by monocytes, in a STING- and inflammasome-dependent manner. Patients suffering from chronic inflammatory disorders (Crohn's disease, rheumatoid arthritis, and chronic hepatitis C virus infection) exhibit high levels of seric IL-26. In addition, IL-26 has been reported in inflammatory and destructive lesions conferring proinflammatory properties to DNA released by dying cells, and setting up a positive amplification loop between extensive cell death and exacerbated inflammation. As a result of DNA cargo IL-26 capacity re-entry of self-DNA likely augments local proinflammatory cytokine secretion, thereby amplifying not only cell recruitment and death but also IL-26 secretion, as a positive feedback loop (Poli et al, 2018).

\section{Strategies in targeting NET/extracellular DNA}

A disproportionate virus-induced NET release can contribute to damage, locally as well as systemically. It will be important to explore the mechanisms that control NET formation in the context of viral infection.

\section{DNase1}

It is widely accepted that naturally-occurring DNase-1 digests extracellular chromatin and NETs (Yang et al, 2020). As such it displays a vital physiological regulation of NETs in the host and the adequate clearance of NETs. Low level bio-activity of endogenous DNase-1 may lead to a dysregulation of NETs, thus causing autoimmune disease and other inflammatory disorders.

Numerous studies have shown, in animal models, that the dismantling of NET DNA fibers by exogenous DNases exerts a protective effect during various pathological situations associated with an excess of NET. DNase is the only NET targeting molecule already being used in clinical practice: the inhalation of recombinant DNase is part of the therapeutic arsenal for cystic fibrosis, improving lung function and reducing infectious exacerbations. DNase-1 was also effective when given 5 minutes after initiation of TRALI where NETs are already present and lung injury is developing.

However, the use of DNase-1 alone against NETs is not without its limitations. Clinical or radiological improvement after DNase-1 treatment of infants with virus-associated bronchiolitis was observed in some clinical trials but not in others (Schonrich et al, 2016)). Besides, the fact that DNase-1 dismantles the NET structure but does not totally degrade the protein components of NETs indicates that it is less effective in abrogating a NET-triggered inflammatory response.

\section{DNase-1 plus histone blockers}

Other therapeutic targets with notable potential include NET components, especially histones. Antihistone antibody infusion, for instance, reduced neutrophil-induced cell damage and lung congestion. Mice were found to be protected from TRALI both by the use of DNase-1 and by the direct targeting of NET components with a histone-blocking antibody. This data suggests that targeting NET formation may be a promising new direction for the treatment of acute lung injury. Caudriller et al targeted NET 
components using a blocking antibody against $\mathrm{H} 2 \mathrm{~A}$ and $\mathrm{H} 4$ histones, and by disrupting the NET scaffold with DNase-1, and found this strategy effective in reducing lung injury and mortality.

\section{Platelet/platelet interactions:}

Aspirin treatment decreased NET formation in the lung microcirculation and plasma, and also decreased lung deposition of platelets with neutrophils on the vascular walls. When platelet-platelet interactions was blocked with a glycoprotein Ilb/IIla inhibitor, mice were also protected from TRALI and mortality (Caudriller et al, 2012).

\section{$\underline{\text { TLR targeting }}$}

Several TLR agonists and antagonists are regarded as promising therapeutic agents for the treatment of sepsis, asthma, vaccine adjuvants, and autoimmunity (Fuchs et al, 2012). Very different structural classes of molecules can inhibit endosomal TLRs by essentially identical mechanisms of action, suggesting a general mechanism for targeting this group of TLRs. AT791 and E6446 may inhibit TLR7 and 9 signaling, to render nucleic acids nonstimulatory, by masking stimulatory sequences and/or altering their conformation (Lamphier et al, 2014). Similarly, the blockade of CLEC5A and TLR2 by the use of a bi-specific anti-CLEC5A/TLR2 monoclonal antibody ( $\mathrm{mAb}$ ) has the potential to reduce the risk of autoimmunity post-DV infection, and may have therapeutic effects on autoimmune diseases by limiting excessive NET formation and persistent inflammasome activation (Fuchs et al, 2012). Two potentially relevant targets downstream of TLR are IRAK4 and IRF5. Efforts are currently underway to block the function of these proteins (Elkon et al, 2019).

AT791 and E6446 share several characteristics with a number of clinically approved lysosomotropic drugs such as haloperidol, levomepromazine, and amantadine. All of these drugs are lipophilic, contain weak bases, exhibit high volumes of distribution in vivo, and have long elimination half-lives. The accumulation of weak bases inside acidic vesicles has the potential to neutralize vesicle $\mathrm{pH}$, and indeed chloroquine, methylamine, and ammonium chloride are commonly used as biologic reagents for this purpose. However, modulation of $\mathrm{pH}$ does not appear to explain the inhibition of TLR7 and 9 signaling either by AT791, E6446, or by the anti-malarials chloroquine or hydroxychloroquine. Hydroxychloroquine (HCQ), a well-applied anti-inflammation drug with TLR-pathway blockage capacity, could effectively abrogate COX2 through activating TLR4/9 (Yang et al, 2020). HCQ has multiple mechanisms of action that may differ according to the pathogen studied (Deveaux et al, 2020). While chloroquine is also used in the treatment of autoimmune diseases (SLE and RA), the activity of the molecule is not limited to malaria and the control of inflammatory processes, as illustrated by its broad-spectrum activity against a range of bacterial, fungal and viral infections.

\section{Inhibitors of cytokines or downstream signaling events}

The use of biologics to block cytokines is now widespread, such as effects of IL- 6 and IL-6 blockade on neutrophil function in vitro and in vivo as observed in RA (Wright et al, 2014). Note, the use of newer small molecule drugs such as 'Jakinibs', introduced to attenuate the biologics' downstream effects (Mehta et al, 2020).

\section{Anti-CLEC5A/TLR2}

NETs contribute to the pathogenesis of autoimmune diseases, where the risk of developing autoimmune diseases is increased in patients post-DV infection (Fuchs et al, 2012). Sung et al (2019) recently demonstrated, blockade of CLEC5A and TLR2 using a bi-specific anti-CLEC5A/TLR2 mAb has the potential to reduce the risk of autoimmunity post-DV infection. Its therapeutic effect on autoimmune diseases, by limiting excessive NET formation and persistent inflammasome activation, was also shown. Alain Interestingly, the release of extracellular vesicles (EVs) induced by the activation of CLEC2 by DV and H5N1 influenza virus (IAV) further enhanced NETosis and proinflammatory cytokine production via CLEC5A and TLR2 (Sung et al, 2019). These findings point toward the immunomodulatory effects of EVs during platelet-leukocyte interactions. 


\section{Other potential NET inhibitors}

Below are other targeting NETs drugs of relevant potential: Surfactant proteins A and D are innate immune proteins in the lungs, whose importance in the clearance of DNA has been demonstrated. The administration of DNase-1 coupled with the neutralization of histones (using specific antibodies or polysialic acid, for example) or administration of proteases would therefore constitute a potential solution in combatting the deleterious effects of NETs. Other new treatments targeting NET formation include Peptidyl arginine deiminase-4 (PAD4) enzyme, which mediates histone citrullination, and may therefore represent a valuable therapeutic approach for septic shock. Given the important mechanism of re-entry of self-DNA allowed by IL26, the possibility of targeting this cytokine must be considered.

\section{Viral-induced ARDS/cytokine storm}

In this section, we focus on the link between cytokine storm and ARDS as they concur to severe illness in the case of COVID.

It is well known that most pro-inflammatory cytokines are released from macrophages. Severe acute infections are usually associated with the activation of macrophages by enveloped viruses such as the DV, H5N1 viruses, and Ebola viruses (Sung et al, 2019). As above indicated, the activation of neutrophils leads to the formation of NETs, which may further aggravate virus-induced inflammatory reactions. It is well established that acute viral infections frequently cause thrombocytopenia, and that plateletleukocyte interactions not only regulate inflammatory reactions but also contribute to the pathogenesis of vascular injury, thrombosis, and autoimmune reaction (Raoult et al, 2020).

Whilst the host immune system is beneficial in promoting the clearance of many pathogens, it can also have adverse effects in certain contexts (Fuchs et al, 2012). One of the most cited examples is the high susceptibility to bacterial infection after acute viral infections, for instance with influenza virus. This may be due to the association of NET formation with lung injury, and the central role of inflammasome activation in viral pathology.

Virus mediates the activation of platelets, resulting in the enhancement of NET formation and proinflammatory cytokine release; it also induces inflammasome activation via membrane receptors, including CLECs. Sung et al (2019) recently showed that the blockade of CLEC5A/TLR2 attenuates NET formation and inflammasome activation, and is thus beneficial to the host during acute viral infection. CLEC5A/TLR2 is therefore critical in microbe-induced NET formation and proinflammatory cytokine production. In particular, these effects are induced by DV and H5N1 influenza virus, by the release of extracellular vesicles that can be regarded as novel DAMP which activate innate immunity via CLEC5A and TLR2.

Respiratory failure from ARDS is the leading cause of COVID-19 associated mortality. Accumulating evidence suggests that a subgroup of patients with severe COVID-19 might have a cytokine storm syndrome. In a recent retrospective, multicentre study of 150 confirmed COVID-19 cases in Wuhan, China, elevated ferritin and IL- 6 were found as predictors of fatality, suggesting that mortality might be due to virally driven hyperinflammation. The massive over-production of cytokines by the host immune system (a phenomenon known as a "cytokine storm") was formerly observed and investigated during the global outburst of SARS-CoV infection in 2003. That virus results in a severe inflammatory reaction and increases systemic vascular permeability occurring within 2 to 5 days following viral exposure.

Almost 200,000 persons in the US develop ALI every year from a variety of causes, including sepsis, bacterial pneumonia, aspiration of gastric contents, and epidemic viruses, such as H1N1 and SARS (Caudriller et al, 2012). NETs have been found in infection-related ALI models of influenza virus, bacteria or bacterial component LPS, and fungi. Substantial inflammation in the alveolar and plasma compartments has been a recognized characteristic of patients with ARDS since the 1980s (as reviewed by Meyer et al, 2017). High concentrations of inflammatory cytokines (cytokine storm) have been associated with both injurious ventilator strategies and poorer outcomes. During a cytokine storm, an 
excessive immune response ravages healthy lung tissue, leading to acute respiratory distress and multi-organ failure. Beyond inflammation, mechanisms including endothelial activation, respiratory epithelial dysfunction, and surfactant depletion are established major contributors to ARDS pathogenesis.

Respiratory failure from ARDS is the leading cause of COVID-19 associated mortality. It has been discovered that the activation of endosomal TLRs (TLR3, TLR7, TLR8, TLR9) and cytosolic sensors by viral nucleic acids induces the production of interferons and proinflammatory cytokines. These intracellular nucleic acid receptors/sensors have been defined as "protective host factors," as they are critical for host defense against viral infections. However, the identity of "pathogenic host factors," and their specific contribution to virus-induced severe inflammatory reactions and lethality remains unclear. Moreover, the origins of distinct clinical symptoms as provoked by different viruses have yet to be fully elucidated.

Indeed the most common critical complications during exacerbation of COVID-19 areARDS, sepsis, acute cardiac injury, heart failure, systemic vascular permeability, disseminated intravascular coagulation and venous thromboembolism. NETosis dysregulation observed in severe cases tends to be characterized by lower lymphocytes counts, higher leukocytes counts, and higher neutrophillymphocyte-ratio (Lagunas-Rangel et al, 2020), as well as lower percentages of monocytes, eosinophils, and basophils (Chuan et al Clin Infect Dis). Biologics such as high levels of IFN, lactate deshydrogenase, $C$ reactive Protein, and fibrinogen are reported in minor cases.

\section{Conclusion}

It is necessary to identify and treat hyperinflammation using existing, approved therapies with known safety profiles, in order to immediately control and reduce the rising COVID-19 mortality (Mehta et al, 2020). The current focus has been on the development of novel therapeutics, including antivirals, immunomodulators and vaccines. The use of a number of specific anti-cytokine approaches to treat a variety of cytokine storm syndromes, including those triggered by viruses, has proven effective. These include drugs targeting IL-1, IL-6, IL-18, and interferon-gamma. The early onset of inflammatory reactions with elevated local or systemic vascular permeability are the common features of SARS-CoV and other acute viral infections prior to the adaptive immune system being fully activated. These observations suggest that innate immune responses contribute significantly to the pathogenesis of acute viral infections (Mehta et al, 2020).

Considering the rapid evolution of the symptoms and the lethality of this disease, we hypothesize that COVID-19 progresses under an uncontrolled amplifier loop leading to hyperinflammation. Refractory patients generally present cytokine storm syndromes sharing the pathology of an overly active immune response, frequently leading to fatal multi-organ dysfunction syndrome, including acute respiratory distress syndrome (ARDS).

We have described here the correlations of COVID-19 symptoms and biological observations with those consecutive to uncontrolled NET formation causing various sterile or infection diseases. General clinical conditions, numerous pathological and biological features are analogous with NET deleterious effects (Table 1). We postulate that COVID-19 induces a disproportionate virus-induced NET release, which plays a key role in COVID-19 pathogenesis.

Viruses are known for their extraordinary capacity to evade immune control mechanisms. We postulate that viral mechanisms target NET formation by impairing the clearance of NET and extracellular DNA. This would lead to harmful positive amplification of virally-driven hyperinflammation. This finding suggests a significant new direction for the development of treatments for this acute viral infection. Investigation of NETosis and the pathogenesis of extracellular 
DNA is all recent, with the result that research and development on treatments that would inhibit the amplifier loop induced by unbalanced NETosis also remains in its infancy. While neutrophils are principally the starting point for DNA release, targeting NETs rather than neutrophils themselves can be a practice strategy.

Two main phases might be considered in OVID-19 progression: when the first symptoms appear, and again at the start of the cytokine storm featuring respiratory failure. While antivirals can be administered throughout the course of the illness, the use of immunomodulators appears less beneficial (and even counterproductive) at the point where the immune response is exacerbated, possibly due to NET's "double-edged sword" effect. A controlled disease should features an early onset of inflammatory reactions with elevated local or systemic vascular permeability, before the adaptive immune system is fully activated, highlighting the significant contribution of the innate immune response. This is the case for a large part of infected patients who experience only mild or no symptoms in the course of the disease. In this light, the COVID-19 evolution may not be unique. Nonetheless, it is obviously a complex disease, as a minority of infected patients suffer severe or lethal conditions, albeit to date apparent coronavirus-2 genomic stability.

We speculate that the cytokine storm, and downstream local and systemic cytotoxicity to endothelial and epithelial walls, result in the impairment of NET and cirDNA. This adverse, self-inflicted pathological effect might trigger an autoimmune-like disease, which would certainly be due to host factors. In that context, it is obviously necessary to investigate of the influence on COVID pathogenesis and NETosis of co-morbidities, individual differences in the immunity state, previous or co-infections, previous diseases, ethnicity, or genetic or epigenetic alterations.

Considering the COVID-19 pandemic and its severe impact on public health, the clinical imperative now should be to implement combination therapy with drugs currently used $n$ patients or in the final stages of clinical development. These drugs should include Remdesivir (GS-5734), a Lopinavir/Ritonavir association (with or without Interferon B-1a), HCQ, anti-IL6, Jakinibs or intravenous immunoglobulin (Mehta et al, 2020). We propose that, in the short term, DNase-1 treatment should be evaluated in clinical trials, with or without associated drugs. We also propose that, in the longer term, a significant increase in research on the development of TLR and CLEC inhibitors, and on anti-IL26 therapies.

Acknowledgements: The authors thank S. Dejasse. A.R. Thierry is supported by INSERM. This workwas funded by the "SIRIC Montpellier Cancer Grant INCa_Inserm_DGOS_12553" 


\section{References}

Al Amir Dache Z, Otandault A, Tanos R, et al. Blood contains circulating cell-free respiratory competent mitochondria. FASEB j. 2020;34(3):3616-3630. doi:10.1096/fj.201901917RR

An J, Woodward JJ, Lai W, et al. Inhibition of Cyclic GMP-AMP Synthase Using a Novel Antimalarial Drug Derivative in Trex1 -Deficient Mice. Arthritis Rheumatol. 2018;70(11):1807-1819. doi:10.1002/art.40559

Bach M, Moon J, Moore R, Pan T, Nelson JL, Lood C. A Neutrophil Activation Biomarker Panel in Prognosis and Monitoring of Patients With Rheumatoid Arthritis. Arthritis Rheumatol. 2020;72(1):4756. doi:10.1002/art.41062

Barrientos L, Bignon A, Gueguen C, et al. Neutrophil Extracellular Traps Downregulate Lipopolysaccharide-Induced Activation of Monocyte-Derived Dendritic Cells. JI. 2014;193(11):56895698. doi:10.4049/jimmunol.1400586

Boudreau LH, Duchez A-C, Cloutier N, et al. Platelets release mitochondria serving as substrate for bactericidal group IIA-secreted phospholipase A2 to promote inflammation. Blood. 2014;124(14):2173-2183. doi:10.1182/blood-2014-05-573543

Cheng OZ, Palaniyar N. NET balancing: a problem in inflammatory lung diseases. Front Immun. 2013;4. doi:10.3389/fimmu.2013.00001

Caudrillier A, Kessenbrock K, Gilliss BM, et al. Platelets induce neutrophil extracellular traps in transfusion-related acute lung injury. J Clin Invest. 2012;122(7):2661-2671. doi:10.1172/JCI61303

D'Abbondanza M, Martorelli EE, Ricci MA, et al. Increased plasmatic NETs by-products in patients in severe obesity. Sci Rep. 2019;9(1):14678. doi:10.1038/s41598-019-51220-x

Devaux CA, Rolain J-M, Colson P, Raoult D. New insights on the antiviral effects of chloroquine against coronavirus: what to expect for COVID-19? International Journal of Antimicrobial Agents. March 2020:105938. doi:10.1016/j.ijantimicag.2020.105938

Fuchs TA, Kremer Hovinga JA, Schatzberg D, Wagner DD, Lämmle B. Circulating DNA and myeloperoxidase indicate disease activity in patients with thrombotic microangiopathies. Blood. 2012;120(6):1157-1164. doi:10.1182/blood-2012-02-412197

Granger V, de Chaisemartin L, Chollet-Martin S. La pêche miraculeuse des filets du neutrophile. Med Sci (Paris). 2014;30(5):544-549. doi:10.1051/medsci/20143005018

Hilscher MB, Shah VH. Neutrophil Extracellular Traps and Liver Disease. Semin Liver Dis. November 2019:s-0039-3399562. doi:10.1055/s-0039-3399562

Hirose T, Hamaguchi S, Matsumoto N, et al. Presence of Neutrophil Extracellular Traps and Citrullinated Histone H3 in the Bloodstream of Critically III Patients. Palaniyar N, ed. PLoS ONE. 2014;9(11):e111755. doi:10.1371/journal.pone.0111755

Lagunas-Rangel FA. Neutrophil-to-lymphocyte ratio and lymphocyte-to-C-reactive protein ratio in patients with severe coronavirus disease 2019 (COVID-19): A meta-analysis. J Med Virol. April 2020. doi:10.1002/jmv.25819 
Lamphier M, Zheng W, Latz E, et al. Novel Small Molecule Inhibitors of TLR7 and TLR9: Mechanism of Action and Efficacy In Vivo. Mol Pharmacol. 2014;85(3):429-440. doi:10.1124/mol.113.089821

Mehta P, McAuley DF, Brown M, Sanchez E, Tattersall RS, Manson JJ. COVID-19: consider cytokine storm syndromes and immunosuppression. The Lancet. 2020;395(10229):1033-1034. doi:10.1016/S0140-6736(20)30628-0

Meyer NJ, Calfee CS. Novel translational approaches to the search for precision therapies for acute respiratory distress syndrome. The Lancet Respiratory Medicine. 2017;5(6):512-523. doi:10.1016/S2213-2600(17)30187-X

Moschonas IC, Tselepis AD. The pathway of neutrophil extracellular traps towards atherosclerosis and thrombosis. Atherosclerosis. 2019;288:9-16. doi:10.1016/j.atherosclerosis.2019.06.919

Moodley M, Moodley J, Naicker T. Neutrophil extracellular traps: The synergy source in the placentae of HIV infected women with pre-eclampsia. Pregnancy Hypertension. 2020;20:69-74.

doi:10.1016/j.preghy.2020.03.007

Nakazawa D, Marschner JA, Platen L, Anders H-J. Extracellular traps in kidney disease. Kidney International. 2018;94(6):1087-1098. doi:10.1016/j.kint.2018.08.035

Pertiwi K, van der Wal A, Pabittei D, et al. Neutrophil Extracellular Traps Participate in All Different Types of Thrombotic and Haemorrhagic Complications of Coronary Atherosclerosis. Thromb Haemost. 2018;118(06):1078-1087. doi:10.1055/s-0038-1641749

Pilsczek FH, Salina D, Poon KKH, et al. A Novel Mechanism of Rapid Nuclear Neutrophil Extracellular Trap Formation in Response to Staphylococcus aureus. JI. 2010;185(12):7413-7425. doi:10.4049/jimmunol.1000675

Poli C, Augusto JF, Dauvé J, et al. IL-26 Confers Proinflammatory Properties to Extracellular DNA. Jl. 2017;198(9):3650-3661. doi:10.4049/jimmunol.1600594

Raoult D, Zumla A, Locatelli F, Ippolito G, Kroemer G. Coronavirus infections: Epidemiological, clinical and immunological features and hypotheses. CST. 2020;4(4):66-75. doi:10.15698/cst2020.04.216

Sanchez C, Snyder MW, Tanos R, Shendure J, Thierry AR. New insights into structural features and optimal detection of circulating tumor DNA determined by single-strand DNA analysis. npj Genomic Med. 2018;3(1):31. doi:10.1038/s41525-018-0069-0

Shida $\mathrm{H}$, Hashimoto N, Kusunoki Y, et al. Anti-neutrophil extracellular trap antibody in a patient with relapse of anti-neutrophil cytoplasmic antibody-associated vasculitis: a case report. BMC Nephrol. 2018;19(1):145. doi:10.1186/s12882-018-0953-y

Schimmel M, Nur E, Biemond BJ, et al. Nucleosomes and neutrophil activation in sickle cell disease painful crisis. Haematologica. 2013;98(11):1797-1803. doi:10.3324/haematol.2013.088021

Schönrich G, Raftery MJ. Neutrophil Extracellular Traps Go Viral. Front Immunol. 2016;7. doi:10.3389/fimmu.2016.00366

Stiel L, Mayeur-Rousse C, Helms J, Meziani F, Mauvieux L. First visualization of circulating neutrophil extracellular traps using cell fluorescence during human septic shock-induced disseminated intravascular coagulation. Thrombosis Research. 2019;183:153-158. doi:10.1016/j.thromres.2019.09.036 
Sung P-S, Hsieh S-L. CLEC2 and CLEC5A: Pathogenic Host Factors in Acute Viral Infections. Front Immunol. 2019;10:2867. doi:10.3389/fimmu.2019.02867

Tan KS, Lim RL, Liu J, et al. Respiratory Viral Infections in Exacerbation of Chronic Airway Inflammatory Diseases: Novel Mechanisms and Insights From the Upper Airway Epithelium. Front Cell Dev Biol. 2020;8:99. doi:10.3389/fcell.2020.00099

Thierry AR, El Messaoudi S, Gahan PB, Anker P, Stroun M. Origins, structures, and functions of circulating DNA in oncology. Cancer Metastasis Rev. 2016;35(3):347-376. doi:10.1007/s10555-0169629-x

Tiku V, Tan M-W, Dikic I. Mitochondrial Functions in Infection and Immunity. Trends in Cell Biology. 2020;30(4):263-275. doi:10.1016/j.tcb.2020.01.006

WHO. Coronavirus disease 2019 (COVID-19) situation report - 52. March 12, 2020. https://www.who.int/docs/default-source/coronaviruse/20200312-sitrep-52-covid-

19.pdf?sfvrsn=e2bfc9c0_2 (accessed March 13, 2020).

Wright HL, Cross AL, Edwards SW, Moots RJ. Effects of IL-6 and IL-6 blockade on neutrophil function in vitro and in vivo. Rheumatology. 2014;53(7):1321-1331. doi:10.1093/rheumatology/keu035

Wu F, Zhao S, Yu B, et al. A new coronavirus associated with human respiratory disease in China. Nature. 2020;579(7798):265-269. doi:10.1038/s41586-020-2008-3

Yang L-Y, Luo Q, Lu L, et al. Increased neutrophil extracellular traps promote metastasis potential of hepatocellular carcinoma via provoking tumorous inflammatory response. J Hematol Oncol. 2020;13(1):3. doi:10.1186/s13045-019-0836-0

Yousefi S, Stojkov D, Germic N, et al. Untangling "NETosis" from NETs. Eur J Immunol. 2019;49(2):221227. doi:10.1002/eji.201747053 


\begin{tabular}{|l|l|}
\hline $\begin{array}{c}\text { Analogous pathological conditions and biological } \\
\text { features when comparing NETosis/extracellular DNA } \\
\text { dysregulation and COVID-19 }\end{array}$ \\
\begin{tabular}{|l} 
Complex disease \\
Inflammatory disease \\
Multi-organ damage
\end{tabular} \\
\hline Pathologies & $\begin{array}{l}\text { Failure of respiratory function } \\
\text { Sepsis } \\
\text { Type } 1 \text { Diabetes sensitzation } \\
\text { ARDS } \\
\text { Pro-thrombic } \\
\text { Abnormality of coagulation function } \\
\text { Systemic vascular permeability } \\
\text { Acute cardiac injury } \\
\text { Heart failure }\end{array}$ \\
\hline High level Neutrophils \\
High level Interferon \\
High level C reactive Protein \\
High level Lactate deshydrogenase \\
High level pro-inflammatory cytokines \\
Elevated presence Fibrinogen
\end{tabular}

Table 1 